\title{
Short sleep and social jetlag are associated with higher intakes of non-milk extrinsic sugars, and social jetlag is associated with lower fibre intakes in those with adequate sleep duration: a cross-sectional analysis from the National Diet and Nutrition Survey Rolling Programme (Years 1-9)
}

\author{
Haya Al Khatib', Vita Dikariyanto', Kate M Bermingham², Rachel Gibson ' and \\ Wendy L Hall ${ }^{1, *}$ \\ 'Department of Nutritional Sciences, School of Life Course and Population Sciences, Faculty of Life Sciences and \\ Medicine, King's College London, London SE 1 9NH, UK: ${ }^{2}$ Department of Twin Research and Genetic Epidemiology, \\ School of Life Course and Population Sciences, Faculty of Life Sciences and Medicine, King's College London, \\ London, UK
}

Submitted 9 July 2021: Final revision received 22 December 2021: Accepted 11 January 2022: First published online 18 January 2022

\begin{abstract}
Objective: To investigate associations and interactions between sleep duration and social jetlag status with nutrient intake, nutrient status, body composition and cardio-metabolic risk factors in a nationally representative UK adult population. Design: A cross-sectional study using 4-d food diary and self-reported sleep data from the UK National Diet and Nutrition Survey Rolling Programme 2008-2017. Setting: UK free-living population.

Subjects: Totally, 5015 adults aged 19-64 years.

Results: Thirty-four per cent were short sleepers ( $<7 \mathrm{~h}$ ); $7 \%$ slept $\geq 9$ h; $14 \%$ had $>2 \mathrm{~h}$ difference in average sleep duration between weeknights and weekend nights (social jetlag). Compared to those reporting optimal sleep duration $(\geq 7-<9 \mathrm{~h})$, short sleep was associated with higher intakes of non-milk extrinsic sugars (NMES) (0.9\% energy, $95 \%$ CI: 0.4, 1.4), total carbohydrate (0.8\% energy, $95 \%$ CI: $0 \cdot 2,1.4)$ and a lower non-starch polysaccharides fibre intake $(-0.5 \mathrm{~g} / \mathrm{d}$, $95 \%$ CI $-0 \cdot 8,-0 \cdot 2)$. There was a significant interaction between short sleep and social jetlag for fibre intakes, where adequate sleepers with social jetlag as well as all short sleepers (regardless of social jetlag) had lower fibre intakes than adequate sleepers with no social jetlag. Short sleep, but not social jetlag, was associated with greater adiposity, but there were no differences in other markers of cardiometabolic disease risk.

Conclusions: The present study reports that both short sleep and social jetlag are associated with higher intakes of NMES, but only sleep duration is associated with markers of adiposity. Social jetlag was associated with lower fibre intakes even in individuals with adequate weekly sleep duration, suggesting catch-up sleep does not prevent the adverse impact of irregular sleep habits on food choices.
\end{abstract}

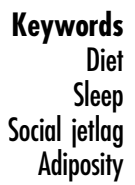

Optimal sleep, determined by duration, number of awakenings, consistency with circadian rhythms and presence/absence of clinical sleep disorders, is vital for optimal health and wellbeing ${ }^{(1)}$. Lack of sleep has been

Haya $\mathrm{Al}$ Khatib and Vita Dikariyanto contributed equally to this work.

Rachel Gibson and Wendy L Hall are joint senior authors. highlighted as a public health concern, particularly as current estimates of sleep duration fall short of the recommended 7-9 h per night for adults ${ }^{(2,3)}$. Self-reported short sleep duration ( $<7 \mathrm{~h}$ per night) has been associated with increased risks of obesity ${ }^{(4,5)}$ and associated cardiometabolic complications ${ }^{(6-10)}$. Long sleep ( $\geq 9 \mathrm{~h}$ ) is also associated with relatively poor health outcomes but represents a

*Corresponding author: Email wendy.hall@kcl.ac.uk

(C) The Author(s), 2022. Published by Cambridge University Press on behalf of The Nutrition Society. This is an Open Access article, distributed under the terms of the Creative Commons Attribution licence (https://creativecommons.org/licenses/by/4.0/), which permits unrestricted re-use, distribution, and reproduction in any medium, provided the original work is properly cited 
potentially complex subpopulation where there may be many important confounding factors ${ }^{(10)}$.

As well as average sleep duration, marked variation in intra-individual sleep timing and sleep duration indicates suboptimal sleep habits and misalignment with circadian rhythms and is implicated in risk of poor health ${ }^{(11,12)}$. The term 'social jetlag' has been coined ${ }^{(13)}$ to capture a habitual pattern of short sleep during working days relative to longer, catch-up sleep, on free days (usually the weekend). Unlike international travel-related jetlag, social jetlag is a lifelong disrupter of optimal sleep habits and has been positively associated with increased $\mathrm{BMI}^{(14)}$ and markers of cardiometabolic disease risk ${ }^{(15)}$.

Proposed mechanisms linking insufficient sleep or circadian disruption with weight gain and metabolic diseases include changes in energy expenditure, hormonal dysregulation and impaired insulin sensitivity ${ }^{(16,17)}$, which may be amplified by modifiable behavioural risk factors ${ }^{(18)}$. As cardiometabolic conditions have strong nutritional determinants, links between poor sleep and metabolic disruption may be mediated through diet ${ }^{(19)}$. Intervention studies under laboratory-controlled conditions have shown that short-term partial sleep deprivation causes higher total energy intakes ${ }^{(20)}$. Chronic exposure to short sleep duration has also been associated with higher energy intakes $^{(21,22)}$, carbohydrate ${ }^{(23)}$, sugar ${ }^{(24)}$ and alcohol intakes $^{(25)}$, as well as lower protein ${ }^{(24)}$, fibre ${ }^{(21)}$ and fruit and vegetable intakes ${ }^{(26)}$, as well as a lower overall diet quality score and unhealthy eating habits ${ }^{(27-29)}$.

Evidence is emerging that dietary patterns and nutrient intakes are also less favourable in those who report social jetlag, including among young university students ${ }^{(30)}$, adolescents ${ }^{(31)}$ and a nationally representative UK adult population (cross-sectional analysis 2008-2011) ${ }^{(32)}$, but it is not clear whether this just reflects chronic sleep deprivation, or whether there is an independent relationship with inconsistency in sleep duration (i.e. social jetlag). The aim of this research is to investigate associations and interactions between sleep duration and social jetlag status with nutrient intake, nutrient status, body composition and cardiometabolic risk factors in a nationally representative UK adult population (2008-2017). The hypothesis was that short sleep duration would be associated with a less healthy dietary profile (higher saturated fat, salt and sugar, lower fibre intakes) and increased adiposity and cardiometabolic risk factors. The second hypothesis was that there would be an interaction between sleep duration and social jetlag.

\section{Methods}

\section{Study design and cobort}

This was a cross-sectional study comparing food intake, nutrient status and cardiometabolic risk factors in short
$(<7 \mathrm{~h})$, adequate $(\geq 7-<9 \mathrm{~h})$ and long sleepers $(\geq 9 \mathrm{~h})$ in the UK National Diet and Nutrition Survey Rolling Programme (NDNS-RP). The NDNS-RP survey consists of a repeated cross-sectional survey that is carried out every 1.5 years with the aim of assessing nutritional intake and status in a nationally representative UK population ${ }^{(33)}$. The present study investigates the combined results from Years 1-9 of the NDNS (2008/2009-2016/2017) and includes adults aged 19-64 years who are not pregnant or lactating. Full details of the methodology have been previously reported elsewhere ${ }^{(34)}$. In summary, a UK representative random sample of individuals was drawn from a list of all the addresses in the UK, the Postcode Address File. Interviewers administered 4-d food diaries, measured participants' weight and height, and assisted participants in completing a computer-assisted personal interview (CAPI) to collect background and lifestyle information, including sleep duration.

\section{Sleep assessment}

Sleep duration questions targeted weekdays and weekends separately, phrased as follows: 'Over the last seven days, that is since (date), how long did you usually sleep for on weeknights. That is Sunday to Thursday nights? and 'Over the last seven days, how long did you usually sleep for on weekend nights. That is Friday and Saturday nights?' Only the time participants were asleep was included. If participants were on a night shift during the previous 2 weeks, then the average time slept during the day was recorded. Shift work and napping habits were not recorded as part of the NDNS-RP. To estimate a 7-d average sleep duration, the following calculation was applied: $((5 \times$ weeknight sleep duration $)+(2 \times$ weekend sleep duration)/7)).

\section{Dietary assessment and analysis}

Participants completed a 4-d estimated food diary with portion sizes were estimated using household measures and weights from labels. To aid accurate estimation, photographs of frequently consumed foods, life-size spoons and a life-size glass were included in the food diary ${ }^{(34)}$. Nutrient intakes were calculated using Diet In Nutrients Out (DINO), a dietary assessment system developed at the Human Nutrition Research Centre at Cambridge University and linked to Public Health England's NDNS Nutrition Databank ${ }^{(34)}$. Dietary intake outcomes included total energy intake, as well as absolute $(\mathrm{g} / \mathrm{d})$ and relative intakes (percentage of total energy intake (\%E)) of the macronutrients and alcohol intakes. Dietary fibre was included as defined by the Englyst ${ }^{(35)}$ method, known as non-starch polysaccharides (NSP). As a proxy measure of carbohydrate quality, the carbohydrate: $\mathrm{NSP}^{(36)}$ ratio was calculated. Dietary misreporters were identified using McCrory's method, using $2 \mathrm{SD}^{(37)}$. 


\section{Nutritional biomarkers}

The 24-h urinary excretion of $\mathrm{Na}, \mathrm{K}$ and $\mathrm{N}$ was analysed as objective biomarkers of dietary intake of salt, fruit and vegetables and protein, respectively ${ }^{(34)}$. Circulating nutritional biomarkers included $\mathrm{Hb}$, plasma ferritin, serum vitamin $\mathrm{B}_{12}$ and plasma vitamin $\mathrm{C}$, which were measured from blood samples collected during the nurse visit which occurred within 2 to 4 months after dietary data collection.

\section{Cardiometabolic risk factors}

\section{Body composition}

Participants' weight and height were measured using a portable weighing scale and stadiometer at the initial interviewer visit. Waist circumference (WC) was measured at the scheduled nurse's visit upon completion of the 4- $\mathrm{d}$ food diary. All measurements were taken twice, and if there were wide variations across measurements (height \pm 0.5 $\mathrm{cm}$, weight $\pm 0.2 \mathrm{~kg}, \mathrm{WC} \pm 3 \mathrm{~cm}$ ), a third was taken and the mean value of the two most similar measurements was reported $^{(34)}$.

\section{Blood pressure}

Participants were asked to refrain from eating, consuming alcohol or exercising vigorously for $30 \mathrm{~min}$ prior to their seated blood pressure measurement. Participants' blood pressure was measured three times at 1-min intervals between readings using an automated blood pressure monitor (Omron HEM907) ${ }^{(34)}$.

\section{Blood measurements}

The present study also evaluates cardiometabolic risk using fasting plasma glucose, glycated $\mathrm{Hb}$ (HbA1c), serum total cholesterol, HDL-cholesterol, LDL-cholesterol, serum TAG and serum C-reactive protein $(\mathrm{CRP})^{(34)}$.

\section{Assessment of confounding variables}

Demographics and lifestyle information were collected using the computer-assisted personal interview CAPI ${ }^{(34)}$. This information incorporates age, sex, economic status, qualifications level, the number of children in the household up to 4 years of age, and frequency of alcohol intake and smoking. Information on having any long-standing illness including cancers, mental illness such as anxiety, as well as endocrine, nutritional or metabolic diseases was also collected.

\section{Statistical analysis}

The present study includes adults aged 19-64 years in the NDNS-RP with sleep duration data available. Sleep duration was divided into three categories to define short $(<7 \mathrm{~h})$, adequate $(\geq 7-<9 \mathrm{~h})$ and long $(\geq 9 \mathrm{~h})$ sleepers as per National Sleep Foundation recommendations ${ }^{(38)}$.

Previous research using the NDNS data set estimated social jet lag as the difference in sleep duration hours between weekend and weeknights, since midpoint sleep data were not available ${ }^{(32)}$. Participants in this study were categorised as having social jetlag if they reported $>2 \mathrm{~h}$ difference in sleep duration between weeknights and weekend nights, based on previous reports showing that this cut-off was associated with increased cardiometabolic risk factors $(14,32,39,40)$

All statistical analyses were performed using IBM SPSS Statistics 25.0 (Statistical Product and Service Solutions, IBM Corp.). Differences in participant characteristics were assessed using chi-square tests and ANOVA for categorical and scale data, respectively. Normal distributions of residuals were visually checked using histograms and Q-Q plots. Log transformation (ln) was conducted for non-normally distributed residuals. The analysis outcomes were back log-transformed into the geometric mean values. The assumption for homoscedasticity was examined by plotting the standardised residuals of dependent variables and predictors. In sensitivity analyses of outcomes of dietary intake, misreporters were excluded, and the analyses were repeated including only plausible reporters.

The associations between sleep duration or social jetlag category and dietary intake (absolute $(\mathrm{g} / \mathrm{d})$ and relative $(\%$ EI)), biomarkers of nutrient status, body composition and cardiometabolic risk factors were assessed using ANCOVA, with Bonferroni corrections for post hoc pairwise comparisons. The model was adjusted for age, sex, ethnicity, BMI (except for when assessing BMI and WC as an outcome), total energy intake (except for when assessing energy intake as an outcome), as well as lifestyle and socio-economic confounders including economic status, whether they currently had a long-standing illness, number of children below 4 years of age, smoking status and alcohol intake (except when assessing alcohol as an outcome). A two-sided $P$-value of $<0.05$ was considered statistically significant. To account for multiple comparisons, we conducted false discovery rate calculation using the Benjamini and Hochberg method ${ }^{(41)}$. Dietary intake categories (e.g. any marker of dietary carbohydrate quality) and/or cardiometabolic health variables that significantly differed by both sleep duration and social jetlag category were selected, and interactions between sleep duration and social jetlag status were included in the model to reveal independent associations.

Chi-square tests were also conducted to assess the association between food groups and social jetlag. For CRP analysis in investigating the difference between a group with and without social jetlag, multinominal logistics regression was used with adjustments for the same factors as above. CRP values were divided into two groups as follows: $<3 \mathrm{mg} / 1$ and $\geq 3-<10 \mathrm{mg} / 1$.

The NDNS-RP requires survey weighting to correct for differences in sample selection and response. The appropriate weights were used for differential selection probabilities of households and individuals, non-response to the individual questionnaires, non-response to the nurse visit and non-response to the blood sample and urine 
collection $^{(34)}$. Weight factor wti_Y19 (weight for individual and diary-all ages, combined Years 1-9 (the UK NDNS-RP 2008-2017)) was the weight factor used for examining differences in dietary intakes among short, adequate and long sleeper groups as well as between a group with and without social jetlag; wtn_Y19 (weight for nurse-all ages, combined Years 1-9 (the UK NDNS-RP 20082017)) was used for investigating differences in body composition variables (BMI and WC), blood pressure and urinary $\mathrm{Na}, \mathrm{K}$ and $\mathrm{N}$; and wtb_Y19 (weight for blood-all ages, combined Years 1-9 (the UK NDNS-RP 2008-2017)) was used for investigating differences in blood analyte variables including CRP, lipids, glucose and biomarkers of nutrient status (vitamin $\mathrm{B}_{12}$, vitamin $\mathrm{C}$, HbA1C, Hb and serum ferritin).

\section{Results}

\section{Participant characteristics}

Sleep data were available for 5015 adults aged 19-64 years (96\% of 5223 total) participating in the NDNS-RP 2008-2017. The characteristics of the cohort in the present study stratified by sleep duration are outlined in Table 1 . Short sleepers comprised $34 \%$ of the cohort, with only $7 \%$ identified as long sleepers. The mean age of the cohort was $42 \cdot 6 \pm 12 \cdot 4$ years, and the mean BMI was $27.5 \pm 5.5 \mathrm{~kg} / \mathrm{m}^{2}$. The cohort was predominantly 'White', and 59\% were female. Two-thirds of the cohort were employed (69\%), and $28 \%$ of participants had obtained a degree or equivalent. Approximately two-thirds $(67 \%)$ of respondents reported not having any chronic illness, and $82 \%$ had no children between the ages of 0 and 4 years of age living in the household. Current smokers consisted of $26 \%$ of the sample, and $38 \%$ reporting consuming no alcohol. A quarter of respondents (26\%) consumed a dietary supplement.

In the unadjusted stratification of the population by sleep status (Table 1), short sleepers were significantly older and had higher BMI than those who slept $\geq 7 \mathrm{~h}$. A greater proportion of long sleepers were female. There were no differences in the ethnic group distributions between sleep groups. More of the longer sleepers were either going to school or college full-time or not working at present, than the shorter and longer sleepers, with the adequate sleepers having the greatest proportion of people in employment. A greater number of short and long sleepers were either current or ex-smokers. Nearly, half of the long sleepers reported consuming no alcohol, with greater proportions of short and adequate sleepers consuming low or moderate amounts of alcohol. Nearly, half of the long sleepers reported having a long-standing illness, with the lowest proportion of those with long-standing illness being reported in adequate sleepers. Short sleep duration was significantly associated with social jetlag, with $19 \cdot 3 \%$ of short sleepers being categorised as having social jetlag, compared to only $11.9 \%$ of adequate sleepers and $7.6 \%$ of long sleepers (Table 1).

In the unadjusted stratification of the population by social jetlag status (Table 1), the social jetlag groups were significantly younger, had a greater proportion of men, and were more likely to be current smokers and drink more than 8 units per $\mathrm{d}$ (men) or 6 units per $\mathrm{d}$ (women) of alcohol than those who had regular sleep duration. BMI and ethnic group distributions did not differ between social jetlag groups. A greater proportion of the social jetlag group were in employment $(80 \cdot 8 \%)$ compared to the no social jetlag group (66.9\%), and there was a slightly larger proportion of the social jetlag group that had no children between the ages of 0 and 4 years old. There were no differences between groups in their reporting of having a long-standing illness.

\section{Dietary energy, macronutrient and micronutrient intakes}

As presented in Table 2, in comparison with adequate sleepers $(\geq 7-<9 \mathrm{~h})$, short sleep $(<7 \mathrm{~h})$ was associated with lower absolute and relative intakes of protein and n-3 PUFA. There were no significant differences in intakes of saturated or trans fats in short sleepers in comparison with adequate sleepers. Short sleep was also associated with higher absolute and relative intake of carbohydrates, non-milk extrinsic sugars (NMES), and a higher carbohydrate:NSP ratio. In addition, short sleep was associated with lower intake of NSP fibre, folate, vitamin $\mathrm{B}_{12}$, vitamin $\mathrm{C}, \mathrm{K}$, $\mathrm{Fe}$, and fruits and vegetables in comparison with adequate sleep. However, biomarkers of intake for vitamin $\mathrm{B}_{12}$, vitamin $\mathrm{C}, \mathrm{Fe}$ (Hb and serum ferritin), and urinary $\mathrm{Na}$ (salt), K and $\mathrm{N}$ (protein) excretion were not significantly different between short sleepers and adequate sleepers (online supplementary material, Supplemental Table 1). In comparison with adequate sleepers $(\geq 7-<9 \mathrm{~h})$, long sleepers ( $\geq 9$ h) had no differences in dietary intakes (Table 2 ).

Results of a sensitivity analysis in plausible dietary intake reporters only ( $85.2 \%$ of the cohort, $n 4274$ ), as presented in Supplemental Table 2, show similar results to the analysis of the whole cohort, including significantly greater NMES intakes and a higher carbohydrate:NSP ratio in short sleepers compared with adequate sleepers. However, unlike the whole sample, both short and long sleepers reported lower energy intakes than adequate sleepers, and there were no differences in total carbohydrate intake (absolute or relative) in plausible reporters. In addition, there was no association between short sleep and $\mathrm{K}$ intake in plausible reporters.

Although short sleep was associated with higher NMES intake, the proportion of consumers of high sugar foods ('sugar confectionery', 'sugars, preserves and sweet spreads', 'soft drinks, not low calorie', 'puddings') was similar between the short sleep and adequate sleep groups (online supplementary material, Supplemental Table 3), 
Table 1 Background characteristics of a representative UK adult population (aged 19-64 years) according to sleep duration and social jetlag status based on NDNS 2008-2019 ( $n$ 5015)

\begin{tabular}{|c|c|c|c|c|c|c|c|c|c|c|}
\hline \multirow[b]{2}{*}{ Characteristics } & & \multicolumn{3}{|c|}{ Sleep duration } & \multirow[b]{2}{*}{$P$-value } & \multirow[b]{2}{*}{$\begin{array}{l}\text { FDR } \\
\text { value }\end{array}$} & \multirow[b]{2}{*}{$\begin{array}{c}\text { No social jetlag ( } n \\
4307)\end{array}$} & \multirow[b]{2}{*}{$\begin{array}{l}\text { Social jetlag }(n \\
708)\end{array}$} & \multirow[b]{2}{*}{$P$-value } & \multirow[b]{2}{*}{$\begin{array}{l}\text { FDR } \\
\text { value }\end{array}$} \\
\hline & & $\begin{array}{c}<7 \mathrm{~h} \\
(n \\
1711) \\
\end{array}$ & $\begin{array}{c}\geq 7-< \\
9 \mathrm{~h} \\
(n \\
2947)\end{array}$ & $\begin{array}{c}\geq 9 \mathrm{~h} \\
(n \\
357)\end{array}$ & & & & & & \\
\hline \multicolumn{11}{|l|}{ Age } \\
\hline Mean & & 45 & 42 & 39 & \multirow[t]{2}{*}{$<0.001$} & \multirow[t]{2}{*}{$<0.001$} & 43 & 39 & \multirow[t]{2}{*}{$<0.001$} & \multirow[t]{2}{*}{$<0.001$} \\
\hline SD & & 12 & 12 & 14 & & & 12 & 12 & & \\
\hline \multirow[t]{2}{*}{ Sex, \% } & Male & $42 \cdot 7$ & 41.4 & $32 \cdot 8$ & \multirow[t]{2}{*}{0.002} & \multirow[t]{2}{*}{0.003} & $40 \cdot 1$ & $48 \cdot 3$ & \multirow[t]{2}{*}{$<0.001$} & \multirow[t]{2}{*}{$<0.001$} \\
\hline & Female & $57 \cdot 3$ & $58 \cdot 6$ & $67 \cdot 2$ & & & 59.9 & $51 \cdot 7$ & & \\
\hline \multicolumn{11}{|l|}{$\mathrm{BMI}, \mathrm{kg} / \mathrm{m}^{2}$} \\
\hline $\begin{array}{l}\text { Mean } \\
\text { SD }\end{array}$ & & $\begin{array}{r}29 \\
6\end{array}$ & $\begin{array}{r}27 \\
5\end{array}$ & $\begin{array}{r}27 \\
6\end{array}$ & $<0.001$ & $<0.001$ & $\begin{array}{r}28 \\
6\end{array}$ & $\begin{array}{r}27 \\
5\end{array}$ & 0.249 & 0.320 \\
\hline \multirow[t]{5}{*}{ Ethnicity, \% } & White & $92 \cdot 3$ & $90 \cdot 7$ & $91 \cdot 8$ & \multirow[t]{5}{*}{0.203} & \multirow[t]{5}{*}{0.203} & 91.3 & $91 \cdot 2$ & \multirow[t]{5}{*}{0.968} & \multirow[t]{5}{*}{0.968} \\
\hline & Mixed ethnic group & 0.9 & $1 \cdot 0$ & $1 \cdot 1$ & & & $1 \cdot 0$ & 1.0 & & \\
\hline & Black or Black British & $2 \cdot 6$ & $2 \cdot 3$ & $2 \cdot 3$ & & & $2 \cdot 5$ & $2 \cdot 1$ & & \\
\hline & Asian or Asian British & 3.5 & $4 \cdot 0$ & $3 \cdot 1$ & & & 3.7 & $4 \cdot 1$ & & \\
\hline & Any other group & 0.7 & 1.9 & $1 \cdot 7$ & & & 1.5 & 1.6 & & \\
\hline \multirow[t]{3}{*}{ Economic status, \% } & $\begin{array}{l}\text { Going to school or college full time (including } \\
\text { on vacation) }\end{array}$ & $2 \cdot 0$ & $3 \cdot 8$ & $6 \cdot 2$ & \multirow[t]{3}{*}{$<0.001$} & \multirow[t]{3}{*}{$<0.001$} & $3 \cdot 1$ & $4 \cdot 8$ & \multirow[t]{3}{*}{$<0.001$} & $<0.001$ \\
\hline & In full- or part-time employment & $67 \cdot 0$ & $72 \cdot 9$ & $44 \cdot 8$ & & & $66 \cdot 9$ & $80 \cdot 8$ & & \\
\hline & Not working at present & $30 \cdot 9$ & 23.4 & $49 \cdot 0$ & & & $30 \cdot 0$ & 14.4 & & \\
\hline Children aged between 0 and 4 & 0 & $81 \cdot 8$ & 81.9 & 81.5 & 0.004 & 0.004 & $81 \cdot 2$ & 85.9 & 0.019 & 0.029 \\
\hline years, \% & 1 & $12 \cdot 4$ & 14.5 & $16 \cdot 0$ & & & $14 \cdot 4$ & $11 \cdot 2$ & & \\
\hline & 2 & $5 \cdot 2$ & $3 \cdot 3$ & $2 \cdot 2$ & & & $4 \cdot 1$ & 2.5 & & \\
\hline & 3 & 0.5 & 0.3 & 0.3 & & & 0.3 & 0.4 & & \\
\hline Smoking status, \% & Current cigarette smoker & $29 \cdot 2$ & 23.4 & 33.2 & $<0.001$ & $<0.001$ & $25 \cdot 0$ & $33 \cdot 0$ & $<0.001$ & $<0.001$ \\
\hline & Ex-regular cigarette smoker & $21 \cdot 3$ & $19 \cdot 8$ & 17.5 & & & $20 \cdot 6$ & 17.5 & & \\
\hline & Never regular cigarette smoker & 49.5 & $56 \cdot 8$ & $49 \cdot 3$ & & & 54.5 & 49.5 & & \\
\hline Alcohol units/d, \% & None & 38.7 & 35.5 & 49.7 & $<0.001$ & $<0.001$ & $38 \cdot 1$ & 34.6 & $0.001^{*}$ & 0.002 \\
\hline & $\leq 4$ (men), $\leq 3$ (women) & $21 \cdot 8$ & 23.7 & 17.5 & & & $22 \cdot 7$ & $21 \cdot 8$ & & \\
\hline & $>4$ and $\leq 8$ (men), $>3$ and $\leq 6$ (women) & $17 \cdot 5$ & $18 \cdot 9$ & $11 \cdot 3$ & & & $18 \cdot 2$ & $16 \cdot 0$ & & \\
\hline & $>8$ (men), > 6 (women) & $21 \cdot 9$ & 21.9 & $21 \cdot 5$ & & & $20 \cdot 9$ & $27 \cdot 6$ & & \\
\hline Long-standing illness present ${ }^{\star}$ & Yes & 37.5 & $29 \cdot 0$ & $47 \cdot 1$ & $<0.001$ & $<0.001$ & 33.4 & $31 \cdot 8$ & 0.425 & 0.478 \\
\hline$\%$ & No & 62.5 & $71 \cdot 0$ & 52.9 & & & $66 \cdot 6$ & $68 \cdot 2$ & & \\
\hline Social jetlag, \% & & $19 \cdot 3$ & 11.9 & $7 \cdot 6$ & $<0.001$ & $<0.001$ & & & & \\
\hline
\end{tabular}

NDNS, Nutrition Survey Rolling Programme; FDR, false discovery rate.

Data are presented as mean \pm SD for continuous variables or $n(\%)$ for categorical variables. Continuous data were tested by one-way ANOVA. $P<0.05$ indicates a significant difference between groups. Multiple comparison-adjusted FDR $P$-values using the Benjamini-Hochberg false discovery rate are also provided. Categorical data were tested by chi-square test. $P<0.05$ indicates a significant association between sleep group and the demographic variables.

${ }^{*}$ Any physical or mental health condition(s) or illness that have lasted, or expected to last, for 12 months or more. 


\section{NS Public Health Nutrition}

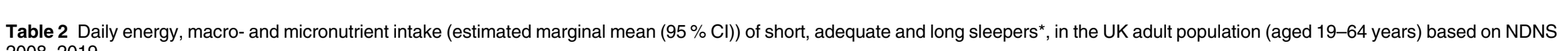
2008-2019

\begin{tabular}{|c|c|c|c|c|c|c|c|c|c|c|}
\hline & \multicolumn{2}{|c|}{ Short sleep, $<7 \mathrm{~h}$} & \multirow[b]{2}{*}{$\begin{array}{l}P \text {-value (short } v \text {. } \\
\text { adequate sleep) }\end{array}$} & \multirow[b]{2}{*}{ FDR value } & \multicolumn{2}{|c|}{ Adequate sleep, $\geq 7-<9 \mathrm{~h}$} & \multicolumn{2}{|c|}{ Long sleep, $\geq 9 \mathrm{~h}$} & \multirow[b]{2}{*}{$\begin{array}{l}P \text {-value (long } v \text {. } \\
\text { adequate sleep) }\end{array}$} & \multirow[b]{2}{*}{$\begin{array}{l}\text { FDR } \\
\text { value }\end{array}$} \\
\hline & $\begin{array}{c}\text { Estimated } \\
\text { marginal mean }\end{array}$ & $95 \% \mathrm{Cl}$ & & & $\begin{array}{c}\text { Estimated } \\
\text { marginal mean }\end{array}$ & $95 \% \mathrm{Cl}$ & $\begin{array}{c}\text { Estimated } \\
\text { marginal mean }\end{array}$ & $95 \% \mathrm{Cl}$ & & \\
\hline $\begin{array}{l}\text { Total energy } \\
\text { (kcal) }\end{array}$ & 1744 & 1657,1831 & $0.026 \ddagger$ & 0.068 & 1791 & 1704,1878 & 1684 & 1579,1788 & $0.004 \ddagger$ & 0.108 \\
\hline Total energy (kJ) & 7337 & 6972,7703 & $0.027 \ddagger$ & 0.068 & 7534 & 7169,7900 & 7084 & 6647,7521 & $0.004 \ddagger$ & 0.108 \\
\hline Protein $(\mathrm{g}) \dagger$ & $68 \cdot 9$ & $66 \cdot 4,71 \cdot 4$ & $<0.001 \ddagger$ & $<0.001 \S$ & 71.6 & $69 \cdot 1,74 \cdot 2$ & $70 \cdot 6$ & $67 \cdot 6,73 \cdot 7$ & 0.929 & 1.000 \\
\hline Protein $(\% \mathrm{EI}) \dagger$ & $16 \cdot 5$ & $15 \cdot 9,17 \cdot 0$ & $<0.001 \ddagger$ & $<0.001 \S$ & $17 \cdot 0$ & $16 \cdot 4,17 \cdot 6$ & $16 \cdot 5$ & $16 \cdot 0,17 \cdot 4$ & 0.552 & 1.000 \\
\hline Fat $(\mathrm{g})$ & $70 \cdot 0$ & $68 \cdot 0,71.9$ & 0.723 & 1.000 & $70 \cdot 4$ & $68.5,72.4$ & 69.4 & $67 \cdot 1,71 \cdot 8$ & 0.525 & 1.000 \\
\hline Fat (\%EI) & 34.4 & $33 \cdot 4,35 \cdot 4$ & 0.369 & 0.710 & $34 \cdot 7$ & $33 \cdot 7,35 \cdot 7$ & 34.1 & $32 \cdot 9,35 \cdot 3$ & 0.405 & 1.000 \\
\hline $\begin{array}{l}\text { Monounsaturated } \\
\text { fat }(\mathrm{g})\end{array}$ & $25 \cdot 9$ & $25 \cdot 0,26 \cdot 8$ & 1.000 & 1.000 & $26 \cdot 0$ & $25 \cdot 1,27 \cdot 0$ & $25 \cdot 9$ & $24 \cdot 8,27 \cdot 0$ & 1.000 & 1.000 \\
\hline $\begin{array}{l}\text { Monounsaturated } \\
\text { fat (\%EI) }\end{array}$ & $12 \cdot 7$ & $12 \cdot 2,13 \cdot 2$ & 0.788 & 1.000 & $12 \cdot 8$ & $12 \cdot 3,13 \cdot 2$ & $12 \cdot 6$ & $12 \cdot 1,13 \cdot 2$ & 1.000 & 1.000 \\
\hline $\begin{array}{l}n-3 \text { fatty acids } \\
(\mathrm{g}) \dagger\end{array}$ & 1.9 & $1 \cdot 8,2 \cdot 0$ & $0.001 \ddagger$ & $0.004 \S$ & $2 \cdot 0$ & $1 \cdot 8,2 \cdot 1$ & 1.9 & $1 \cdot 7,2 \cdot 0$ & $0 \cdot 196$ & 1.000 \\
\hline $\begin{array}{l}n-3 \text { fatty acids (\% } \\
\text { EI) }\end{array}$ & 1.0 & $1 \cdot 0,1 \cdot 1$ & $0.008 \ddagger$ & $0.024 \S$ & $1 \cdot 1$ & $1 \cdot 0,1 \cdot 1$ & $1 \cdot 0$ & $0.9,1 \cdot 1$ & $0 \cdot 219$ & 1.000 \\
\hline$n-6$ fatty acids $(\mathrm{g})$ & $10 \cdot 5$ & $10 \cdot 0,11 \cdot 0$ & 0.368 & 0.710 & $10 \cdot 7$ & $10 \cdot 2,11 \cdot 2$ & $10 \cdot 7$ & $10 \cdot 1,11 \cdot 3$ & 1.000 & 1.000 \\
\hline $\begin{array}{l}n-6 \text { fatty acids (\% } \\
\text { EI) }\end{array}$ & $5 \cdot 2$ & $5 \cdot 0,5 \cdot 5$ & 0.055 & 0.125 & $5 \cdot 4$ & $5 \cdot 1,5 \cdot 6$ & $5 \cdot 3$ & $5 \cdot 0,5 \cdot 6$ & 1.000 & 1.000 \\
\hline Saturated fat $(\mathrm{g})$ & $25 \cdot 0$ & $24 \cdot 0,26 \cdot 1$ & 1.000 & 1.000 & $25 \cdot 0$ & $24 \cdot 0,26 \cdot 1$ & 24.4 & $23 \cdot 1,25 \cdot 6$ & 0.296 & 1.000 \\
\hline $\begin{array}{l}\text { Saturated fat (\% } \\
\text { EI) }\end{array}$ & $12 \cdot 2$ & $11 \cdot 7,12 \cdot 7$ & 1.000 & 1.000 & $12 \cdot 2$ & $11 \cdot 7,12 \cdot 7$ & 11.9 & $11 \cdot 3,12 \cdot 6$ & 0.446 & 1.000 \\
\hline Trans fat $(\mathrm{g}) \dagger$ & 1.0 & $0.9,1.1$ & 0.477 & 0.883 & 1.0 & $0.9,1.1$ & 1.0 & $0.9,1.1$ & 0.071 & 0.888 \\
\hline Trans fat $(\% \mathrm{EI}) \dagger$ & 0.5 & $0.5,0.6$ & 0.830 & 1.000 & 0.5 & $0.5,0.6$ & 0.5 & $0.5,0.6$ & 0.183 & 1.000 \\
\hline Carbohydrate (g) & 227.5 & $222 \cdot 1,233 \cdot 0$ & $0.017 \ddagger$ & $0.047 \S$ & 224.4 & $219 \cdot 0,229 \cdot 8$ & 227.9 & $221 \cdot 3,234.4$ & 0.288 & 1.000 \\
\hline $\begin{array}{l}\text { Carbohydrate (\% } \\
\text { EI) }\end{array}$ & 48.9 & $47 \cdot 8,50 \cdot 1$ & $0.002 \ddagger$ & $0.008 \S$ & $48 \cdot 1$ & $47 \cdot 0,49 \cdot 3$ & $49 \cdot 0$ & $47 \cdot 7,50 \cdot 4$ & 0.116 & 1.000 \\
\hline NMES (g) & $57 \cdot 0$ & $52 \cdot 0,61 \cdot 9$ & $<0.001 \ddagger$ & $<0.001 \S$ & $52 \cdot 3$ & $47 \cdot 3,57 \cdot 3$ & $53 \cdot 8$ & $47 \cdot 8,59 \cdot 7$ & 1.000 & 1.000 \\
\hline NMES (\%EI) & 11.9 & $10 \cdot 8,12 \cdot 9$ & $<0.001 \ddagger$ & $<0.001 \S$ & 11.0 & $9 \cdot 9,12 \cdot 0$ & $11 \cdot 3$ & $10 \cdot 1,12 \cdot 6$ & 1.000 & 1.000 \\
\hline Alcohol (g) & $10 \cdot 2$ & $7 \cdot 0,12 \cdot 4$ & 0.743 & 1.000 & $9 \cdot 4$ & $6 \cdot 2,12 \cdot 6$ & 8.5 & $4 \cdot 7,12 \cdot 4$ & 1.000 & 1.000 \\
\hline Alcohol (\%El) & $3 \cdot 1$ & $2 \cdot 1,4 \cdot 2$ & 1.000 & 1.000 & 3.0 & $2 \cdot 0,4 \cdot 0$ & $2 \cdot 7$ & $1.5,3.9$ & 1.000 & 1.000 \\
\hline $\operatorname{NSP}(g) \dagger$ & $12 \cdot 6$ & $12 \cdot 0,13 \cdot 3$ & $<0.001 \ddagger$ & $0.002 \S$ & $13 \cdot 1$ & $12 \cdot 5,13 \cdot 8$ & $13 \cdot 2$ & $12 \cdot 4,13.9$ & 1.000 & 1.000 \\
\hline $\begin{array}{l}\text { Carbohydrate: } \\
\text { NSP† }\end{array}$ & $17 \cdot 2$ & $16 \cdot 3,18 \cdot 0$ & $<0.001 \ddagger$ & $<0.001 \S$ & $16 \cdot 4$ & $15 \cdot 6,17 \cdot 3$ & $16 \cdot 8$ & $15 \cdot 8,17 \cdot 8$ & 0.815 & 1.000 \\
\hline Folate $(\mu \mathrm{g}) \dagger$ & $220 \cdot 1$ & $208 \cdot 5,232 \cdot 3$ & $0.001 \ddagger$ & $0.004 \S$ & 229.3 & $217 \cdot 0,242 \cdot 0$ & 237.7 & $222 \cdot 7,253 \cdot 7$ & 0.243 & 1.000 \\
\hline Vitamin $B_{12}(\mu \mathrm{g}) \dagger$ & 4.3 & $4 \cdot 0,4 \cdot 7$ & $0.002 \ddagger$ & $0.008 \S$ & 4.6 & $4 \cdot 2,4.9$ & 4.4 & $4.0,4.9$ & 1.000 & 1.000 \\
\hline
\end{tabular}


or higher in the adequate sleep group ('buns, cakes, pastries and fruit pies', 'chocolate confectionery'). However, a greater proportion of adequate sleepers consumed 'brown, granary and wheatgerm bread', 'cheese', 'eggs and egg dishes', 'fruit', 'fruit juice', 'nuts and seeds', 'oily fish', 'other white fish, shellfish and fish dishes', 'salad and other raw vegetables', 'savoury sauces, pickles, gravies, and condiments', 'semi-skimmed milk', 'vegetables, not raw' and 'wine' compared to short and long sleep groups.

As presented in Table 3, there were no significant differences in intakes of energy, protein, fat, total carbohydrate or alcohol between those with social jetlag compared with no social jetlag. However, social jetlag was associated with higher absolute NMES intakes and lower relative intakes of SFA, and absolute and relative intakes of trans fatty acids. There were no differences in NSP fibre or carbohydrate:NSP ratio, nor any differences in vitamin and mineral intakes, fruits and vegetables intake (Table 3), nor biomarkers of salt, $\mathrm{K}$, protein, Fe, vitamin $\mathrm{C}$ and vitamin $\mathrm{B}_{12}$ intake (online supplementary material, Supplemental Table 4).

A greater proportion of the social jetlag group reported consuming 'chocolate confectionery', 'crisps and savoury snacks' and 'other meat and meat products' (online supplementary material, Supplemental Table 5). A smaller proportion of the social jetlag group consumed 'fruit', 'oily fish', 'other potatoes, potato salads, dishes' (i.e. not including potato chips, fried roast potatoes and potato products'), and 'tea, coffee and water' compared to those not reporting social jetlag.

Short sleep was associated with a higher BMI and WC in comparison with the adequate sleep group (Table 4), but there were no differences between groups for blood pressure, fasting glucose concentrations, HbA1c, blood lipids or CRP. There were no differences in any of the cardiometabolic risk factors between the long sleep and adequate sleep groups. Body composition was not associated with social jetlag status, and there were no other differences in markers of cardiometabolic risk in the social jetlag group compared with the no social jetlag group.

\section{Sleep duration and social jetlag interactions}

Since NMES (g) intakes were higher in the short sleep and social jetlag groups, and NSP intakes were lower in short sleepers only, interactions between sleep duration and social jetlag were investigated for indicators of carbohydrate quality. There was a significant sleep duration $x$ social jetlag interaction for NSP intake $(P=0.022)$. Geometric means (95\% CI) for NSP fibre in adequate sleepers were significantly higher in those with no social jetlag $(13 \cdot 2 \mathrm{~g} / \mathrm{d}(12 \cdot 5,13 \cdot 8), n 2596)$ compared those with social jetlag $(12.7 \mathrm{~g} / \mathrm{d}(12.0,13.5), n 351, P=0.043)$, whereas fibre intake was lower overall in short sleepers compared with adequate sleepers with no social jetlag 
Table 3 Daily energy, macro- and micronutrient intake (estimated marginal mean $(95 \% \mathrm{Cl})$ ) of a group with and without social jetlag*, in the UK adult population (aged 19-64 years) based on NDNS 2008-2019

\begin{tabular}{|c|c|c|c|c|c|c|}
\hline \multirow[b]{2}{*}{ Dietary intake } & \multicolumn{2}{|c|}{ No social jetlag } & \multicolumn{2}{|c|}{ Social jetlag } & \multirow[b]{2}{*}{$P$-value } & \multirow[b]{2}{*}{ FDR value } \\
\hline & Estimated marginal mean & $95 \% \mathrm{Cl}$ & Estimated marginal mean & $95 \% \mathrm{Cl}$ & & \\
\hline Total energy (kcal) & 1762 & 1676,1848 & 1757 & 1663,1851 & 0.832 & 0.961 \\
\hline Total energy (kJ) & 7412 & 7052,7771 & 7393 & 6998,7788 & 0.853 & 0.961 \\
\hline Protein $(\mathrm{g}) \dagger$ & $70 \cdot 2$ & $67 \cdot 8,72 \cdot 8$ & $70 \cdot 0$ & $67 \cdot 3,72 \cdot 8$ & 0.733 & 0.961 \\
\hline Protein $(\% \mathrm{El}) \dagger$ & $16 \cdot \overline{7}$ & $16 \cdot 2,17 \cdot 3$ & $16 \cdot 7$ & $16 \cdot 1,17 \cdot 4$ & 0.901 & 0.961 \\
\hline Fat $(\mathrm{g})$ & $70 \cdot 3$ & $68 \cdot 4,72 \cdot 2$ & 69.4 & $67 \cdot 2,71 \cdot 5$ & 0.082 & 0.241 \\
\hline Fat $(\% \mathrm{El})$ & $34 \cdot 6$ & $33 \cdot 6,35 \cdot 6$ & 33.9 & $32 \cdot 8,35 \cdot 0$ & $0.011 \ddagger$ & 0.081 \\
\hline Monounsaturated fat (g) & $26 \cdot 0$ & $25 \cdot 1,26 \cdot 9$ & $26 \cdot 0$ & $25 \cdot 0,27 \cdot 0$ & 0.866 & 0.961 \\
\hline $\begin{array}{l}\text { Monounsaturated } \\
\text { fat }(\% \mathrm{EI})\end{array}$ & $12 \cdot 7$ & $12 \cdot 3,13 \cdot 2$ & $12 \cdot 6$ & $12 \cdot 1,13 \cdot 1$ & 0.553 & 0.961 \\
\hline$n-3$ fatty acids $(\mathrm{g}) \dagger$ & 1.93 & $1.81,2.05$ & 1.86 & $1.73,1.99$ & $0.039 \ddagger$ & 0.178 \\
\hline$n-3$ fatty acids (\%EI) & $1 \cdot 1$ & $1 \cdot 0,1 \cdot 1$ & $1 \cdot 0$ & $0.9,1.1$ & 0.054 & 0.193 \\
\hline$n-6$ fatty acids $(\mathrm{g})$ & $10 \cdot 6$ & $10 \cdot 1,11 \cdot 1$ & $10 \cdot 5$ & $10 \cdot 0,11 \cdot 1$ & 0.725 & 0.961 \\
\hline$n-6$ fatty acids (\%EI) & $5 \cdot 3$ & $5 \cdot 1,5 \cdot 6$ & $5 \cdot 3$ & $5 \cdot 0,5 \cdot 6$ & 0.637 & 0.961 \\
\hline Saturated fat $(g)$ & $25 \cdot 1$ & $24 \cdot 1,26 \cdot 1$ & 24.5 & $23 \cdot 4,25 \cdot 6$ & $0.035 \ddagger$ & $0 \cdot 177$ \\
\hline Saturated fat (\%EI) & $12 \cdot 3$ & $11 \cdot 8,12 \cdot 8$ & 11.9 & $11 \cdot 3,12 \cdot 4$ & $0.003 \ddagger$ & $0.041 \S$ \\
\hline Trans fat $(\mathrm{g}) \dagger$ & 1.04 & $0.96,1.12$ & 0.96 & $0.88,1.04$ & $<0.001 \ddagger$ & $0.002 \S$ \\
\hline Trans fat $(\% \mathrm{EI}) \dagger$ & 0.53 & $0.49,0.57$ & 0.49 & $0.45,0.53$ & $<0.001 \ddagger$ & $0.002 \S$ \\
\hline Carbohydrate (g) & $225 \cdot 7$ & $220 \cdot 3,231.0$ & $228 \cdot 7$ & $222 \cdot 8,234 \cdot 5$ & $0.044 \ddagger$ & 0.178 \\
\hline Carbohydrate (\%EI) & 48.5 & $47 \cdot 4,49 \cdot 6$ & $49 \cdot 1$ & $47 \cdot 9,50 \cdot 4$ & $0.033 \ddagger$ & 0.177 \\
\hline NMES $(\mathrm{g})$ & 54.0 & $49 \cdot 1,58 \cdot 9$ & $58 \cdot 3$ & $53 \cdot 0,63 \cdot 7$ & $0.001 \ddagger$ & $0.022 \S$ \\
\hline NMES (\%EI) & $11 \cdot 3$ & $10 \cdot 3,12 \cdot 3$ & $12 \cdot 1$ & $11 \cdot 0,13 \cdot 2$ & $0.006 \ddagger$ & 0.063 \\
\hline Alcohol (g) & $10 \cdot 0$ & $6 \cdot 9,13 \cdot 2$ & 8.0 & $4.6,11.5$ & $0.023 \ddagger$ & 0.146 \\
\hline Alcohol (\%El) & $3 \cdot 1$ & $2 \cdot 1,4 \cdot 1$ & 2.6 & $1.5,3.7$ & 0.071 & 0.237 \\
\hline NSP fibre $(\mathrm{g}) \dagger$ & $12 \cdot 9$ & $12 \cdot 3,13 \cdot 5$ & $12 \cdot 7$ & $12 \cdot 1,13 \cdot 4$ & 0.350 & 0.729 \\
\hline Carbohydrate:NSP (g)† & $16 \cdot 7$ & $15 \cdot 9,17 \cdot 6$ & $17 \cdot 1$ & $16 \cdot 2,18 \cdot 1$ & 0.080 & 0.241 \\
\hline Folate $(\mu \mathrm{g}) \dagger$ & 225.9 & $214 \cdot 0,238 \cdot 2$ & $222 \cdot 5$ & $209 \cdot 8,235 \cdot 8$ & 0.311 & 0.707 \\
\hline Vitamin $B_{12}(\mu \mathrm{g}) \dagger$ & 4.4 & $4 \cdot 1,4.8$ & 4.4 & $4 \cdot 0,4 \cdot 8$ & 0.697 & 0.961 \\
\hline Vitamin C (mg)† & $67 \cdot 7$ & $60 \cdot 9,75.2$ & $67 \cdot 6$ & $60 \cdot 3,75 \cdot 9$ & 0.966 & 0.978 \\
\hline $\mathrm{K}(\mathrm{mg}) \dagger$ & $2547 \cdot 8$ & $2460 \cdot 2,2641 \cdot 2$ & $2540 \cdot 2$ & $2443 \cdot 0,2641 \cdot 2$ & 0.700 & 0.961 \\
\hline $\mathrm{Ca}(\mathrm{mg}) \dagger$ & 675.2 & $646 \cdot 1,706 \cdot 3$ & 673.8 & $641.6,707 \cdot 7$ & 0.891 & 0.961 \\
\hline $\mathrm{Fe}(\mathrm{mg}) \dagger$ & 9.9 & $9 \cdot 5,10 \cdot 3$ & 9.7 & $9 \cdot 3,10 \cdot 2$ & 0.115 & 0.303 \\
\hline Fruit and vegetables $(\mathrm{g}) \dagger$ & 253.2 & $227 \cdot 1,281.9$ & 246.9 & $219 \cdot 1,277 \cdot 9$ & 0.394 & 0.788 \\
\hline
\end{tabular}

NDNS, Nutrition Survey Rolling Programme; FDR, false discovery rate; \%El, \% energy intake; NMES, non-milk extrinsic sugars; NSP, non-starch polysaccharides. ANCOVA was used adjusted for age, sex, BMI, total energy intake (except for when assessing energy intake as the outcome), ethnicity, economic status, smoking status, alcohol intake (except when assessing alcohol as an outcome), number of children below 4 years of age and long-standing illness.

*Without social jetlag $n$ 3638; with social jetlag $n 617$.

†Residual data are non-normally distributed. The values presented from these variables are geometric (back log-transformed) means with $95 \% \mathrm{Cl}$.

$\ddagger$ Two-sided $P<0.05$ shows a significant difference.

$\S$ Significant difference using multiple comparison-adjusted FDR $P$-values using the Benjamini-Hochberg false discovery rate.

but did not differ within the short sleep group according to social jetlag category (no social jetlag $12 \cdot 6 \mathrm{~g} / \mathrm{d}(12 \cdot 0,13 \cdot 2)$, $n 1381$; social jetlag $12 \cdot 9 \mathrm{~g} / \mathrm{d}(12 \cdot 2,13 \cdot 6), n 330, P=0 \cdot 221)$. There were no significant interactions between sleep duration and social jetlag for total carbohydrate, NMES or carbohydrate:NSP ratio.

\section{Discussion}

This cross-sectional study examined whether both sleep duration and social jetlag were associated with dietary intakes and cardiometabolic risk factors in a representative sample of UK adults, and further investigated whether there were independent effects of social jetlag regardless of sleep duration. Consistent with previous evidence ${ }^{(28,42)}$, we report that approximately one-third of the UK adult population are not achieving recommended sleep durations, and $14 \%$ were experiencing social jetlag, as defined by a difference in weeknight and weekend night sleep duration $<2 \mathrm{~h}$. As expected, we found a higher prevalence of social jetlag in short sleepers in comparison with those achieving adequate sleep ${ }^{(32)}$.

Nutrient intakes in short sleepers suggested a less healthy diet, characterised by higher intake of added sugars and lower intake of NSP fibre, $n$ - 3 fatty acids, protein, fruits and vegetables, and specific micronutrients, compared with adequate sleepers. A previous analysis of the NDNS-RP years 1-4 reporting on linear associations of dietary intake and sleep duration without adjustment for total energy intake found no significant associations between sleep and nutrient intakes ${ }^{(43)}$, which might be explained by the relationship between sleep and diet being non-linear ${ }^{(19)}$. Whilst habitual sleep deficiency may 
Table 4 Cardiometabolic risk (estimated marginal mean $(95 \% \mathrm{CI})$ ) in a representative UK adult population (aged 19-64 years)* according to sleep duration and social jetlag status based on NDNS 2008-2019

\begin{tabular}{|c|c|c|c|c|c|c|c|c|c|c|c|c|c|c|c|c|}
\hline \multirow[b]{2}{*}{$\begin{array}{l}\text { Cardiometabolic } \\
\text { risk factor }\end{array}$} & \multicolumn{2}{|c|}{ Short sleep, $<7 \mathrm{~h}$} & \multirow{2}{*}{$\begin{array}{c}P \text {-value } \\
\text { (short } v \text {. } \\
\text { adequate } \\
\text { sleep) }\end{array}$} & \multirow[b]{2}{*}{$\begin{array}{l}\text { FDR } \\
\text { value }\end{array}$} & \multicolumn{2}{|c|}{$\begin{array}{l}\text { Adequate sleep, } \geq 7-< \\
\qquad 9 \mathrm{~h}\end{array}$} & \multicolumn{2}{|c|}{ Long sleep, $\geq 9 \mathrm{~h}$} & \multirow{2}{*}{$\begin{array}{c}P \text {-value } \\
\text { (long } v \text {. } \\
\text { adequate } \\
\text { sleep) }\end{array}$} & \multirow[b]{2}{*}{$\begin{array}{l}\text { FDR } \\
\text { value }\end{array}$} & \multicolumn{2}{|c|}{ No social jetlag } & \multicolumn{2}{|c|}{ Social jetlag } & \multirow[b]{2}{*}{$\begin{array}{c}P- \\
\text { value }\end{array}$} & \multirow[b]{2}{*}{$\begin{array}{l}\text { FDR } \\
\text { value }\end{array}$} \\
\hline & $\begin{array}{c}\text { Estimated } \\
\text { marginal } \\
\text { mean }\end{array}$ & $95 \% \mathrm{Cl}$ & & & $\begin{array}{c}\text { Estimated } \\
\text { marginal } \\
\text { mean }\end{array}$ & $95 \% \mathrm{Cl}$ & $\begin{array}{c}\text { Estimated } \\
\text { marginal } \\
\text { mean }\end{array}$ & $95 \% \mathrm{Cl}$ & & & $\begin{array}{c}\text { Estimated } \\
\text { marginal } \\
\text { mean }\end{array}$ & $95 \% \mathrm{Cl}$ & $\begin{array}{l}\text { Estimated } \\
\text { marginal } \\
\text { mean }\end{array}$ & $95 \% \mathrm{Cl}$ & & \\
\hline BMI (kg/m²) & 28.4 & $27 \cdot 4,29 \cdot 4$ & $<0.001 \ddagger$ & $<0.001 \|$ & $27 \cdot 1$ & $26 \cdot 2,28 \cdot 1$ & $26 \cdot 8$ & $25 \cdot 6,27 \cdot 9$ & 0.982 & 1.000 & 27.7 & $26 \cdot 7,28 \cdot 6$ & $27 \cdot 8$ & $26 \cdot 7,28 \cdot 8$ & 0.602 & 0.961 \\
\hline$W C(\mathrm{~cm})$ & 93.9 & $90.3,97.5$ & $0.005 \ddagger$ & $0.017 \|$ & 91.6 & $88 \cdot 0,95 \cdot 2$ & 89.5 & $85 \cdot 2,93.9$ & 0.401 & 1.000 & 94.3 & $92 \cdot 0,96 \cdot 7$ & 95.1 & $92 \cdot 6,97 \cdot 6$ & 0.231 & 0.550 \\
\hline $\mathrm{SBP}(\mathrm{mmHg})$ & $120 \cdot 4$ & $117 \cdot 8,123 \cdot 0$ & 0.062 & 0.135 & 121.8 & $119 \cdot 2,124 \cdot 4$ & 123.5 & $120 \cdot 3,126 \cdot 7$ & 0.361 & 1.000 & $121 \cdot 3$ & $118 \cdot 8,123.9$ & $121 \cdot 2$ & $118 \cdot 3,124 \cdot 0$ & 0.851 & 0.961 \\
\hline $\mathrm{DBP}(\mathrm{mmHg})$ & 73.3 & $71 \cdot 3,75 \cdot 3$ & 1.000 & 1.000 & 73.5 & $71 \cdot 5,75 \cdot 5$ & 74.8 & $72 \cdot 4,77 \cdot 3$ & 0.325 & 1.000 & 73.5 & $71 \cdot 5,75 \cdot 4$ & 73.6 & $71 \cdot 5,75 \cdot 8$ & 0.786 & 0.961 \\
\hline $\begin{array}{l}\text { Glucose } \\
(\mathrm{mmol} / \mathrm{l})\end{array}$ & $5 \cdot 2$ & $4.9,5.5$ & 0.649 & 1.000 & $5 \cdot 2$ & $4 \cdot 9,5 \cdot 5$ & $5 \cdot 2$ & $4 \cdot 8,5 \cdot 5$ & 1.000 & 1.000 & $5 \cdot 2$ & $5 \cdot 0,5 \cdot 4$ & $5 \cdot 2$ & $4 \cdot 9,5 \cdot 4$ & 0.895 & 0.961 \\
\hline $\mathrm{HbA1C}(\%) \dagger$ & 5.6 & $5 \cdot 4,5 \cdot 7$ & $0.038 \ddagger$ & 0.091 & 5.5 & $5 \cdot 4,5 \cdot 7$ & $5 \cdot 6$ & $5 \cdot 4,5 \cdot 8$ & 0.145 & 1.000 & 5.5 & $5 \cdot 4,5 \cdot 7$ & 5.5 & $5 \cdot 4,5 \cdot 7$ & 0.926 & 0.965 \\
\hline $\mathrm{TC}(\mathrm{mmol} / \mathrm{l})$ & 5.0 & $4 \cdot 7,5 \cdot 3$ & 1.000 & 1.000 & $5 \cdot 0$ & $4 \cdot 7,5 \cdot 3$ & 4.9 & $4 \cdot 6,5 \cdot 3$ & 1.000 & 1.000 & $5 \cdot 0$ & $4 \cdot 7,5 \cdot 3$ & 4.9 & $4 \cdot 6,5 \cdot 2$ & $0.046 \ddagger$ & 0.178 \\
\hline TAG $(\mathrm{mmol} / \mathrm{l}) \dagger$ & $1 \cdot 1$ & $1 \cdot 0,1 \cdot 3$ & 1.000 & 1.000 & $1 \cdot 1$ & $1 \cdot 0,1 \cdot 3$ & $1 \cdot 1$ & $1 \cdot 0,1 \cdot 3$ & 1.000 & 1.000 & $1 \cdot 1$ & $1 \cdot 0,1 \cdot 3$ & $1 \cdot 1$ & $1 \cdot 0,1 \cdot 3$ & 0.903 & 0.961 \\
\hline $\mathrm{HDL}(\mathrm{mmol} / \mathrm{l})$ & 1.5 & $1.4,1 \cdot 6$ & 1.000 & 1.000 & 1.5 & $1 \cdot 3,1 \cdot 6$ & 1.5 & $1 \cdot 3,1 \cdot 6$ & 1.000 & 1.000 & 1.5 & $1.4,1 \cdot 6$ & 1.4 & $1.3,1.5$ & $0.009 \ddagger$ & 0.078 \\
\hline LDL (mmol/l) & 3.0 & $2 \cdot 7,3 \cdot 2$ & 1.000 & 1.000 & 3.0 & $2 \cdot 7,3 \cdot 2$ & $2 \cdot 9$ & $2 \cdot 7,3.2$ & 1.000 & 1.000 & 2.9 & $2 \cdot 6,3 \cdot 1$ & $2 \cdot 8$ & $2 \cdot 6,3 \cdot 1$ & 0.443 & 0.852 \\
\hline TC:HDL† & 3.4 & $3 \cdot 2,3 \cdot 7$ & 1.000 & 1.000 & 3.4 & $3.1,3.7$ & 3.4 & $3.1,3.7$ & 1.000 & 1.000 & 3.4 & $3 \cdot 2,3 \cdot 7$ & 3.5 & $3 \cdot 2,3 \cdot 8$ & 0.325 & 0.707 \\
\hline CRP $(\mathrm{mg} / \mathrm{l}) \dagger, \S$ & 1.7 & $1 \cdot 4,2 \cdot 1$ & 1.000 & 1.000 & 1.7 & $1 \cdot 4,2 \cdot 1$ & 1.7 & $1 \cdot 5,2 \cdot 3$ & 1.000 & 1.000 & 1.7 & $1 \cdot 4,2 \cdot 1$ & 1.6 & $1 \cdot 3,1.9$ & 0.978 & 0.978 \\
\hline
\end{tabular}

NDNS, Nutrition Survey Rolling Programme; FDR, false discovery rate; WC, waist circumference; SBP, systolic blood pressure; DBP, diastolic blood pressure; HbA1C, glycated Hb; TC, total cholesterol; CRP, C-reactive protein.

*BMI: short, adequate and long sleepers $n$ 1082, 1930 and 215, respectively; without social jetlag $n 2755 ; n$ with social jetlag $n 472$. WC: short, adequate, and long sleepers $n$ 869, 1594 and 178, respectively; without social jetlag $n 2824$; with

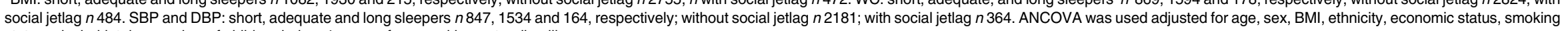
status, alcohol intake, number of children below 4 years of age and long-standing illness.

Glucose: short, adequate and long sleepers $n 708,1279$ and 125 , respectively; without social jetlag $n 1821$; with social jetlag $n 291$. HbA1C: short, adequate and long sleepers $n 715$, 1294 and 134 , respectively; without social jetlag $n 1835$; with social jetlag $n 308$. TC, HDL and TC:HDL: short, adequate and long sleepers $n 737,1350$ and 134, respectively; without social jetlag $n$ 1908; with social jetlag $n 313$. TAG: short, adequate and long sleepers $n 735,1348$ and 134, respectively; without social jetlag $n 1905$; with social jetlag $n 312$. LDL: short, adequate and long sleepers $n 728,1326$ and 134, respectively; without social jetlag $n 1882$; with social jetlag $n 306$. CRP: short, adequate and long sleepers $n 738,1350$ and 134,

†Residual data are non-normally distributed. The values presented from these variables are geometric (back log-transformed) means with $95 \% \mathrm{Cl}$

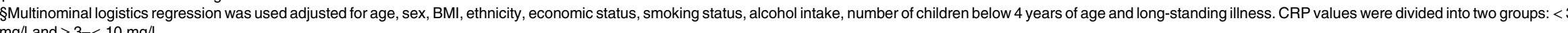

|lSignificant difference using multiple comparison-adjusted FDR P-values using the Benjamini-Hochberg false discovery rate. 
promote hedonic cravings for sweet, energy-dense foods resulting in poorer health outcomes, populations with average sleep durations $>9 \mathrm{~h}$ might sleep longer because of long-standing health conditions, thus confounding associations with diet.

Short sleep was associated with higher carbohydrate intakes and poorer quality carbohydrate intake, evidenced by higher absolute and relative intake of NMES and a higher carbohydrate:NSP ratio, which may lead to greater risk of weight gain. Indeed, short sleep was associated with higher adiposity measures, in agreement with a meta-analysis of twelve prospective cohort studies that followed up from 1 to 12 years showing that short sleep duration was associated with greater risk of obesity ${ }^{(44)}$. Short sleepers may be less physically active ${ }^{(45)}$ leading to a net positive energy balance. However, it could have also been mediated by higher consumption of energy-dense, fibre-poor foods. Sleep-deprived subjects had alterations in neuronal responses in areas of the brain linked to reward, which would be expected to result in a heightened preference for energy-dense, palatable foods ${ }^{(46,47)}$, alongside increased ghrelin secretion ${ }^{(47,48)}$. Therefore, extending sleep duration could reduce cravings for energy-dense, low-fibre foods, and in fact we observed a decreased intake in free sugars following a sleep extension intervention in short sleepers ${ }^{(49)}$. Alongside previous observational evidence $^{(21,23,24)}$, it appears likely that higher dietary sugars and low dietary fibre are the key aspects of diet quality that may predispose to weight gain when sleep duration is habitually inadequate.

Social jetlag was also associated with higher sugar intakes. However, the social jetlag group differed from short sleepers in that they also had lower relative mean intakes of saturated and trans fatty acids and had none of the other significant differences in dietary intake nor cardiometabolic risk factors. A significant interaction between sleep duration and social jetlag revealed that NSP fibre intake was not modified by social jetlag status in short sleepers, but that intakes were lower in adequate sleepers with social jetlag than those without social jetlag. This suggests that both short sleep and sleep inconsistency between week and weekend nights may influence dietary carbohydrate quality independently, although it is also possible that reverse causation was at work and lower fibre intakes might somehow reduce sleep duration.

There are some limitations in this cross-sectional study. Temporal trends cannot be ascertained and no causal inferences or directions of effect can be drawn. Measures of sleep duration and dietary intake rely on self-report, which may be inaccurate. The difference in sleep duration between weeknights and weekend nights was used as a proxy measure for social jet lag, a method applied in observational studies when sleep-onset and wake-up data are not available to calculate difference in sleep midpoint between 'work nights' and 'free nights'(32). Social jetlag was calculated from the self-reported difference in sleep durations between the typical working weeknights and weekend nights, but not all participants would have worked Monday to Friday with weekends off ${ }^{(13)}$. No information was available on shift work status and duration or hours of work; these may implicate circadian disruption via different mechanisms. In individuals not currently in employment, retirement status was not considered, nor menopausal status in women. Finally, later time of eating and a longer eating window have been associated with increased cardiometabolic risk and may impact on sleep duration $^{(50)}$. The present study did not investigate these additional dietary variables but should be a consideration for future research.

\section{Conclusion}

In conclusion, the present study reports that short sleep and social jetlag are associated with higher intake of sugar in a representative UK population sample. Furthermore, the association between short sleep and low fibre intake is extended to those with social jetlag even if they have adequate average weekly sleep duration, suggesting that catch-up sleep does not mitigate the effects of irregular sleep durations on diet quality. This study corroborates previous findings that short sleepers exhibit higher markers of adiposity but found no independent effects of social jetlag on body composition in the adjusted analysis. Combined with increasingly sedentary behaviours of the modern lifestyles, poor dietary carbohydrate quality in short sleepers and those with social jetlag may increase the risk of weight gain and cardiometabolic diseases over time. Prospective cohort studies utilising nutritional biomarkers of carbohydrate quality and objective measures of sleep are needed to address the potential lifelong impact of modest circadian misalignments associated with modern working lifestyles, which affect a large proportion of the global population.

\section{Acknowledgements}

Acknowledgements: This paper uses data from the National Diet and Nutrition Survey (NDNS) Rolling Programme. The NDNS is supported by Public Health England and the UK Food Standards Agency. Financial support: This research received no specific grant from any funding agency, commercial or not-for-profit sectors. Conflict of interest: H.A.K. has been an employee of Zoe Global Ltd. All other authors have none to declare. Authorship: The authors' contributions were as follows: W.L.H. and H.A.K. conceived the idea for the research; V.D., W.L.H., H.A.K. and R.G. designed the research; V.D. and K.M.B. analysed the data; H.A.K. advised on analysis of the data; H.A.K. and V.D. wrote the initial draft manuscript; R.G. and W.L.H. conducted critical revisions and editing; all authors read, 
commented and approved the final manuscript; W.L.H. had primary responsibility for final content. Ethics of human subject participation: This study was conducted according to the guidelines laid down in the Declaration of Helsinki, and all procedures involving research study participants were approved by the National Health Service Local Research Ethics Committee covering each of the postal sectors. Written informed consent was obtained from all participants.

\section{Supplementary material}

For supplementary material/s referred to in this article, please visit https://doi.org/10.1017/S1368980022000167

\section{References}

1. Luyster FS, Strollo PJ, Zee PC et al. (2012) Sleep: a health imperative. Sleep 35, 727-734.

2. Zhu G, Catt M, Cassidy S et al. (2019) Objective sleep assessment in $>80000$ UK mid-life adults: associations with sociodemographic characteristics, physical activity and caffeine. PLOS ONE 14, e0226220.

3. Sheehan CM, Frochen SE, Walsemann KM et al. (2019) Are U.S. adults reporting less sleep? Findings from sleep duration trends in the National Health Interview Survey, 2004-2017. Sleep J 42, 1-8.

4. Cappuccio FP, Taggart FM, Kandala N-B et al. (2008) Metaanalysis of short sleep duration and obesity in children and adults. Sleep 31, 619-626.

5. Zhou Q, Zhang M \& Hu D (2019) Dose-response association between sleep duration and obesity risk: a systematic review and meta-analysis of prospective cohort studies. Sleep Breath 23, 1035-1045.

6. Cappuccio FP, Cooper D, D'Elia Let al. (2011) Sleep duration predicts cardiovascular outcomes: a systematic review and meta-analysis of prospective studies. Eur Heart J 32, 14841492.

7. Wang Q, Xi B, Liu M et al. (2012) Short sleep duration is associated with hypertension risk among adults: a systematic review and meta-analysis. Hypertens Res 35, 1012-1018.

8. Xi B, He D, Zhang M et al. (2014) Short sleep duration predicts risk of metabolic syndrome: a systematic review and meta-analysis. Sleep Med Rev 18, 293-297.

9. Shan Z, Ma H, Xie M et al. (2015) Sleep duration and risk of type 2 diabetes: a meta-analysis of prospective studies. Diabetes Care 38, 529-537.

10. Chaput J-P, Dutil C, Featherstone R et al. (2020) Sleep duration and health in adults: an overview of systematic reviews. Appl Physiol Nutr Metab 45, S218-S231.

11. Patel SR, Hayes AL, Blackwell T et al. (2014) The association between sleep patterns and obesity in older adults. Int J Obes 38, 1159-1164.

12. Bei B, Wiley JF, Trinder J et al. (2016) Beyond the mean: a systematic review on the correlates of daily intraindividual variability of sleep/wake patterns. Sleep Med Rev 28, 108124.

13. Wittmann M, Dinich J, Merrow M et al. (2006) Social jetlag: misalignment of biological and social time. Chronobiol Int 23, 497-509.

14. Roenneberg T, Allebrandt KV, Merrow M et al. (2012) Social jetlag and obesity. Curr Biol 22, 939-943.
15. Parsons MJ, Moffitt TE, Gregory AM et al. (2015) Social jetlag, obesity and metabolic disorder: investigation in a cohort study. Int J Obes 39, 842-848.

16. Killick R, Hoyos CM, Melehan KL et al. (2015) Metabolic and hormonal effects of 'catch-up' sleep in men with chronic, repetitive, lifestyle-driven sleep restriction. Clin Endocrinol 83, 498-507.

17. McHill AW \& Wright KP (2017) Role of sleep and circadian disruption on energy expenditure and in metabolic predisposition to human obesity and metabolic disease. Obes Rev 18, $15-24$.

18. Grandner MA, Seixas A, Shetty S et al. (2016) Sleep duration and diabetes risk: population trends and potential mechanisms. Curr Diab Rep 16, 106.

19. Dashti HS, Scheer FA, Jacques PF et al. (2015) Short sleep duration and dietary intake: epidemiologic evidence, mechanisms, and health implications. Adv Nutr 6, 648-659.

20. Al Khatib HK, Harding SV, Darzi J et al. (2017) The effects of partial sleep deprivation on energy balance: a systematic review and meta-analysis. Eur J Clin Nutr 71, 614-624.

21. Grandner MA, Jackson N, Gerstner JR et al. (2013) Dietary nutrients associated with short and long sleep duration. Data from a nationally representative sample. Appetite $\mathbf{6 4}$, 71-80.

22. Dashti HS, Zuurbier LA, de Jonge E et al. (2016) Actigraphic sleep fragmentation, efficiency and duration associate with dietary intake in the Rotterdam Study. J Sleep Res 25, 404-411.

23. Martinez SM, Tschann JM, Butte NF et al. (2017) Short sleep duration is associated with eating more carbohydrates and less dietary fat in mexican american children. Sleep $\mathbf{4 0}$, zsw057.

24. Kant AK \& Graubard BI (2014) Association of self-reported sleep duration with eating behaviors of American adults: NHANES 2005-2010. Am J Clin Nutr 100, 938-947.

25. Galli G, Piaggi P, Mattingly MS et al. (2013) Inverse relationship of food and alcohol intake to sleep measures in obesity. Nutr Diabetes 3, e58.

26. G囚bski J, Jezewska-Zychowicz M, Guzek D et al. (2018) The associations between dietary patterns and short sleep duration in Polish Adults (LifeStyle Study). Int JEnviron Res Public Health 15, 2497.

27. Theorell-Haglöw J, Lemming EW, Michaëlsson $\mathrm{K}$ et al. (2020) Sleep duration is associated with healthy diet scores and meal patterns: results from the population-based EpiHealth study. J Clin Sleep Med 16, 9-18.

28. Jansen EC, Prather A \& Leung CW (2020) Associations between sleep duration and dietary quality: results from a nationally-representative survey of US adults. Appetite 153, 104748.

29. Córdova FV, Barja S \& Brockmann PE (2018) Consequences of short sleep duration on the dietary intake in children: a systematic review and metanalysis. Sleep Med Rev 42, 68-84.

30. Zerón-Rugerio MF, Cambras T \& Izquierdo-Pulido M (2019) Social jet lag associates negatively with the adherence to the Mediterranean Diet and Body Mass Index among young adults. Nutrients 11, 1756

31. Cetiner O, Yildirim G \& Kalyoncu ZB (2021) Social jetlag is associated with the frequency of consumption of sugarsweetened beverages and a high BMI percentile in adolescents: results of the cross-sectional Family Life, Activity, Sun, Health, and Eating (FLASHE) Study. J Acad Nutr Diet 121, 1721-1731.e1.

32. Almoosawi S, Palla L, Walshe I et al. (2018) Long sleep duration and social jetlag are associated inversely with a healthy dietary pattern in adults: results from the UK National Diet and Nutrition Survey Rolling Programme Y1-4. Nutrients 10, 1131.

33. Department of Health and Social Care, Food Standards Agency \& Public Health England (2019) National Diet and Nutrition Survey. Years 1 to 9 of the Rolling Programme 
(2008/2009-2016/2017): Time Trend and Income Analyses. https://assets.publishing.service.gov.uk/government/uploads/ system/uploads/attachment_data/file/772434/NDNS_UK_Y19_report.pdf (accessed June 2021).

34. Department of Health and Social Care, Food Standards Agency \& Public Health England (2019) NDNS: Results from Years 9-11 (Combined) - Appendices. https://www.gov.uk/ government/statistics/ndns-results-from-years-9-to-11-2016to-2017-and-2018-to-2019 (accessed June 2021).

35. Englyst HN, Quigley ME \& Hudson GJ (1994) Determination of dietary fibre as non-starch polysaccharides with gas-liquid chromatographic, high-performance liquid chromatographic or spectrophotometric measurement of constituent sugars. Analyst 119, 1497-1509.

36. Lloyd-Jones DM, Hong Y, Labarthe D et al. (2010) Defining and setting national goals for cardiovascular health promotion and disease reduction. Circulation 121, 586-613.

37. McCrory MA, Hajduk CL \& Roberts SB (2002) Procedures for screening out inaccurate reports of dietary energy intake. Public Health Nutr 5, 873-882.

38. Hirshkowitz M, Whiton K, Albert SM et al. (2015) National sleep foundation's updated sleep duration recommendations: final report. Sleep Heal 1, 233-243.

39. Rutters F, Lemmens SG, Adam TC et al. (2014) Is social jetlag associated with an adverse endocrine, behavioral, and cardiovascular risk profile? J Biol Rhythms 29, 377-383.

40. Koopman ADM, Rauh SP, van 't Riet E et al. (2017) The association between social jetlag, the metabolic syndrome, and type 2 diabetes mellitus in the general population: the new Hoorn study. J Biol Rhythms 32, 359-368.

41. Benjamini Y \& Hochberg Y (1995) Controlling the false discovery rate: a practical and powerful approach to multiple testing. J R Stat Soc Ser B 57, 289-300.
42. Fatima Y, Bucks RS, Mamun AA et al. (2020) Sleep trajectories and mediators of poor sleep: findings from the longitudinal analysis of 41094 participants of the UK Biobank cohort. Sleep Med 76, 120-127.

43. Potter GDM, Cade JE \& Hardie LJ (2017) Longer sleep is associated with lower BMI and favorable metabolic profiles in UK adults: findings from the National Diet and Nutrition Survey. PLOS ONE 12, e0182195.

44. Bacaro V, Ballesio A, Cerolini S et al. (2020) Sleep duration and obesity in adulthood: an updated systematic review and meta-analysis. Obes Res Clin Pract 14, 301-309.

45. Bromley LE, Booth JN, Kilkus JM et al. (2012) Sleep restriction decreases the physical activity of adults at risk for type 2 diabetes. Sleep 35, 977-984.

46. St-Onge M-P, Wolfe S, Sy M et al. (2014) Sleep restriction increases the neuronal response to unhealthy food in normal-weight individuals. Int J Obes 38, 411-416.

47. Rihm JS, Menz MM, Schultz H et al. (2019) Sleep deprivation selectively upregulates an amygdala-hypothalamic circuit involved in food reward. J Neurosci 39, 888-899.

48. Lin J, Jiang Y, Wang G et al. (2020) Associations of short sleep duration with appetite-regulating hormones and adipokines: a systematic review and meta-analysis. Obes Rev 21, e13051.

49. Al Khatib HK, Hall WL, Creedon A et al. (2018) Sleep extension is a feasible lifestyle intervention in free-living adults who are habitually short sleepers: a potential strategy for decreasing intake of free sugars? A randomized controlled pilot study. Am J Clin Nutr 107, 43-53.

50. Dashti HS, Gómez-Abellán P, Qian J et al. (2021) Late eating is associated with cardiometabolic risk traits, obesogenic behaviors, and impaired weight loss. Am J Clin Nutr 113, 154-161. 